\title{
Cardiac Lead Dislodgement Requiring Reposition/Reoperation
}

National Cancer Institute

\section{Source}

National Cancer Institute. Cardiac Lead Dislodgement Requiring Reposition/Reoperation. NCI Thesaurus. Code C100034.

The movement (macroscopic or microscopic) of an existing lead within the heart or vascular tree away from the original implantation site. (ACC) 